\title{
Anomalous layering at the liquid Sn surface
}

\author{
Oleg G. Shpyrko, ${ }^{1}$ Alexei Yu. Grigoriev, ${ }^{2}$ Christoph Steimer, ${ }^{2}$ Peter S. Pershan,,${ }^{1,2}$ \\ Binhua Lin, ${ }^{3}$ Mati Meron, ${ }^{3}$ Tim Graber, ${ }^{3}$ Jeff Gebhardt, ${ }^{3}$ Ben Ocko, ${ }^{4}$ and Moshe Deutsch ${ }^{5}$ \\ ${ }^{1}$ Department of Physics, Harvard University, Cambridge Massachusetts 02138 (USA) \\ ${ }^{2}$ Division of Engineering and Applied Sciences, Harvard University, Cambridge Massachusetts 02138 (USA) \\ ${ }^{3}$ The Center for Advanced Radiation Sources, University of Chicago, Chicago, Illinois 60637 (USA) \\ ${ }^{4}$ Department of Physics, Brookhaven National Lab, Upton New York 11973 (USA) \\ ${ }^{5}$ Department of Physics, Bar-Ilan University, Ramat-Gan 52900 (Israel)
}

(Dated: June 27, 2018)

\begin{abstract}
X-ray reflectivity measurements on the free surface of liquid Sn are presented. They exhibit the high-angle peak, indicative of surface-induced layering, also found for other pure liquid metals (Hg, Ga, and In). However, a low-angle shoulder, not hitherto observed for any pure liquid metal, is also found, indicating the presence of a high-density surface layer. Fluorescence and resonant reflectivity measurements rule out the assignment of this layer to surface segregation of impurities. The reflectivity is modeled well by a $10 \%$ contraction of the spacing between the first and second atomic surface layers, relative to that of subsequent layers. Possible reasons for this are discussed.
\end{abstract}

PACS numbers: 68.10.--m, 61.10.-i

\section{INTRODUCTION}

Rice and co-workers ${ }^{1}$ predicted that the atoms at the free surface of a liquid metal should be stratified to a depth of a few atomic diameters. This layering phenomenon was experimentally confirmed two decades later by x-ray reflectivity measurements for three high-surfacetension metals: $\mathrm{Hg}_{2}^{2} \mathrm{Ga}^{3}$, and $\mathrm{In}, \stackrel{4}{\underline{4}}$ and the low-surfacetension metal $K \stackrel{5}{5}$ The signature of layering in the $\mathrm{x}$ ray reflectivity curve is the appearance of a quasi-Bragg peak at a wave vector transfer $q_{z}=2 \pi / d$, where $d$ is the atomic spacing between the layers. The peak arises from constructive interference of waves diffracted by the ordered surface layers. These measurements are complicated by several technical issues, in particular the need to use UHV conditions to preserve surface cleanliness for highly reactive liquid metals surfaces.

Both $\mathrm{Ga}$ and In were found to exhibit a simple layering structure described in detail by Regan et $a l^{3}{ }^{3}$ comprising equal-density, periodically spaced atomic layers. Liquid $\mathrm{Hg}^{2.6}$ shows a more complicated surface structure. However, unlike $\mathrm{Ga}$ and $\mathrm{In}$, the high vapor pressure of $\mathrm{Hg}$ did not allow its study under UHV conditions, and the possibility that the more complicated structure originates in chemical interactions with foreign atoms at the surface cannot be definitively ruled out. For Ga, in particular, the decay length of the layering into the bulk was found to be slightly larger than that expected, and found, for other liquid metals. This was tentatively assigned to the enhanced Ga-Ga pairing tendency, ${ }_{3}^{3}$ reflected also in the appearance of a small shoulder on the first, nearestneighbor, peak of the bulk radial distribution function,, .8 and in the ordering of $\mathrm{Ga}$ at the liquid-solid interface ${ }^{9} \mathrm{~A}$ similarly strong pairing tendency has been reported also for $\mathrm{Sn}$, the subject of the present study 10.11 .12

The motivation of the present study was to investigate whether atomic layering exists in liquid Sn and, if it does, to find out whether or not the layering follows the classic behavior of $\mathrm{Ga}$ and In or do new effects appear, e.g. due to the strong pairing. The relatively low melting temperature, $T_{m}=232{ }^{\circ} \mathrm{C}$ and vanishingly low vapor pressure at $T_{m}$ render such measurements possible. As shown below, the measured x-ray reflectivity of the Sn surface indeed exhibits an anomalous feature that is not present for either Ga or In. Additional experiments were carried out to rule out the possibility that this anomalous feature is not intrinsic, but caused by chemical impurities at the surface. The origin of this structure is identified as a $\sim 10 \%$ reduction in the first to second interlayer distance.

\section{EXPERIMENT}

The UHV chamber and the preparation procedures of the liquid Sn sample have been described previously 4.13 Prior to placing an Sn sample inside the Mo sample pan, the surface of the pan was sputtered clean. In the sputtering process the sample's surface is bombarded by $\mathrm{Ar}^{+}$ ions, which break up and sputter away the oxidized material covering the surface. Ingots of solid Sn with purity of 99.9999\% were placed in the Mo sample pan inside a UHV chamber evacuated to $10^{-9}$ Torr and were then melted by a Boralectric heating element mounted underneath the pan. After the molten Sn filled the pan, forming approximately 10 -mm-thick liquid sample, it was cooled to the solid phase, followed by a 24 -h bakeout process. During such bakeout the walls of the chamber and its components are gradually heated up to $100-200^{\circ} \mathrm{C}$ and then cooled down to room temperature. Following this procedure the sample was melted again and the macroscopic native oxide, as well as any possible contaminations, at the surface were removed by a mechanical scraping of the liquid surface with Mo foil wiper. After this, the residual microscopic surface oxide layer was removed by further sputtering by the $\mathrm{Ar}^{+}$ion beam for several hours. 


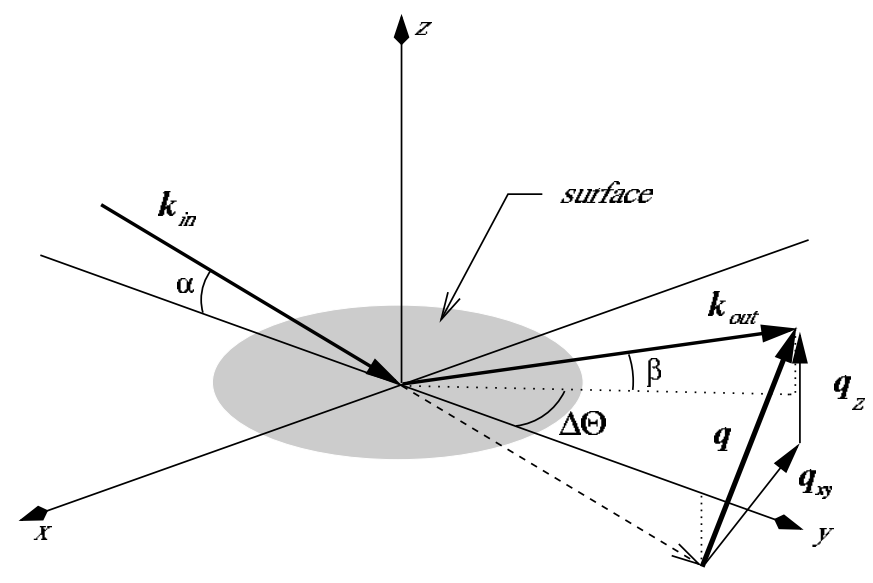

FIG. 1: Kinematics of the x-ray measurement. $k_{\text {in }}$ and $k_{\text {out }}$ are the wave vectors of the incident and detected $\mathrm{x}$-rays, respectively.

If there are impurities in the bulk with surface energies that are lower than that of Sn the Gibbs adsorption rule implies that they would segregate at the surface. We will demonstrate below that even though Sn has a relatively high surface tension impurities are not present at the surface.

\section{X-RAY REFLECTIVITY MEASUREMENTS}

The measurements were carried out at the liquid surface spectrometer facility of the ChemMat CARS beamline at the Advanced Photon Source, Argonne National Laboratory, Argonne, IL. The kinematics of x-ray measurements described in this work are illustrated in Fig. [1] $\mathrm{X}$ rays with a wave vector $k_{i n}=2 \pi / \lambda$, where $\lambda=0.729 \AA$ is the $\mathrm{x}$-ray wavelength, are incident on the horizontal liquid surface at an angle $\alpha$. The detector selects, in general, a ray with an outgoing wave vector $k_{\text {out }}$. The reflectivity $R\left(q_{z}\right)$ is the intensity ratio of these two rays, when the specular conditions $\alpha=\beta$ and $\Delta \Theta=0$ are fulfilled. In this case the surface-normal momentum transfer is $q_{z}=(2 \pi / \lambda)(\sin \alpha+\sin \beta)=(4 \pi / \lambda) \sin \alpha$. The detector resolution due to a finite acceptance angle was defined by two pairs of slits mounted on the detector arm and during all specular reflectivity measurements was fixed at $3.9 \mathrm{mrad}$ vertically and $2.6 \mathrm{mrad}$ horizontally. During the measurements the temperature of the sample has been maintained at $240^{\circ} \mathrm{C}$, which is just above bulk melting temperature of Sn, $232^{\circ} C$.

The x-ray specular reflectivity shown with circles in Fig. 2 is the difference between the specular signal recorded with an Oxford scintillation detector at $\alpha=\beta, \Delta \Theta=0$ and the off-specular background signal recorded at the same $q_{z}$, but at $\Delta \Theta= \pm 0.1^{\circ}$. The data are then normalized to the measured incident intensity. This background subtraction procedure is particularly important because the bulk scattering function peaks at

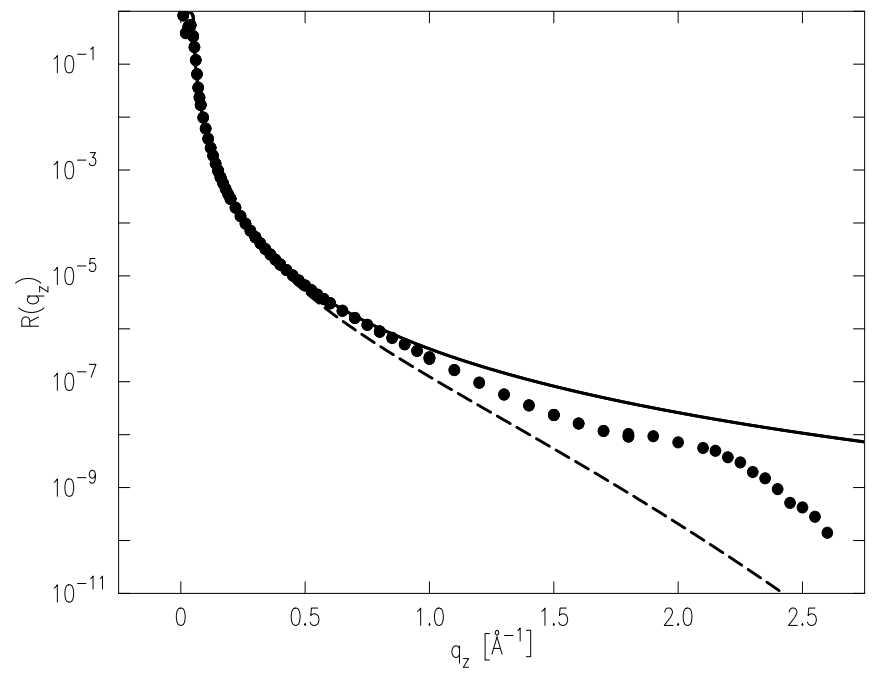

FIG. 2: The measured x-ray specular reflectivity (points) of the surface of liquid Sn. The Fresnel reflectivity (solid line) of an ideally flat and abrupt surface and the reflectivity of an ideal surface roughened by thermally excited capillary waves (dashed line) are also shown.

approximately the same $q_{z}$ as the quasi-Bragg surface layering peak, since both peaks correspond roughly to the interatomic distance. As a result the background is particularly strong at the $q_{z}$ values corresponding to the layering peak. Since the measured $\Delta \Theta \neq 0$ intensity includes contributions from the capillary-wave-induced diffuse surface scattering, all of the theoretical simulations discussed below include a similar background subtraction procedure. The Fresnel reflectivity curve $R_{F}\left(q_{z}\right)$, due to an ideally flat and abrupt surface, is shown as a solid line in Fig. 2] The dashed line is $R_{F}\left(q_{z}\right)$ modified by the theoretically predicted thermal capillary wave contributions, which depend only on the known values of the resolution function, surface tension and temperature. The quasi-Bragg layering peak and the deviation of the measured $R\left(q_{z}\right)$ from the capillary-wave-modified $R_{F}\left(q_{z}\right)$ are unambiguous proof of the existence of local structure at the surface. The quasi-Bragg peak at $q_{z} \approx 2.2 \AA^{-1}$ corresponds to an atomic layering of close-packed spheres with the $d \approx 2.8 \AA$ spacing of the atomic diameter of Sn. An additional new feature, a subtle but significant shoulder at $q_{z} \approx 0.9 \AA^{-1}$ is not discernible in this figure and is revealed only upon removal of the effects of the capillary waves from the measured Fresnel-normalized reflectivity, as we show below.

\section{SURFACE STRUCTURE FACTOR}

To obtain a quantitative measure of the intrinsic surface structure factor the effects of thermal capillary excitations must be deconvolved from the reflectivity curve shown in Fig. 2 As mentioned above, the method for doing this has been described in detail in earlier papers 


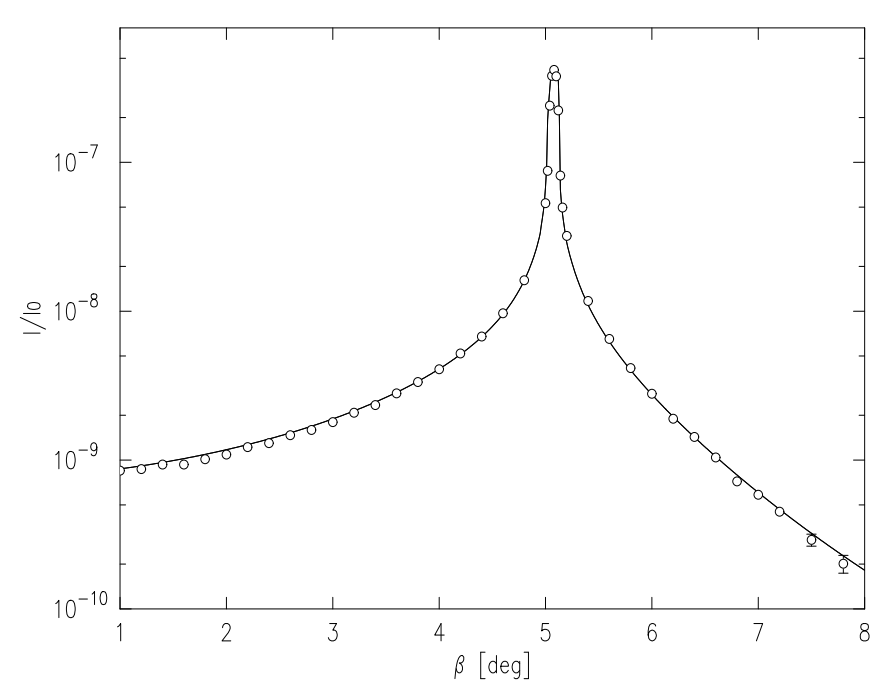

FIG. 3: Diffuse scattering off the liquid Sn surface measured at a fixed grazing angle of incidence $\alpha=5.05^{\circ}$ (open circles). The peak corresponds to the specular condition at $q_{z}=0.48 \AA^{-1}$. The line is the capillary wave theory predictions for surface tension $\gamma=560 \mathrm{mN} / \mathrm{m}$.

on the surface layering in $\mathrm{Ga}, \stackrel{3}{\underline{3}} \mathrm{In}, \frac{4}{\underline{4}} \mathrm{~K}^{5}$, and water $\stackrel{14}{\underline{14}}$ The principal result of this analysis is that the measured reflectivity can be expressed as

$$
R\left(q_{z}\right)=R_{F}\left(q_{z}\right) \cdot\left|\Phi\left(q_{z}\right)\right|^{2} \cdot C W\left(q_{z}\right)
$$

where $\Phi\left(q_{z}\right)$ is the intrinsic structure factor of the surface and $\mathrm{CW}\left(q_{z}\right)$ accounts for the effects of the thermally excited capillary waves on $R\left(q_{z}\right)$. The surface scattering cross section yielding the $\mathrm{CW}\left(q_{z}\right)$ term depends on $T, q_{z}$, the surface tension and the geometric parameters defining the reflectometer's resolution function. It is understood well enough to allow us to fully account analytically for the effects of capillary waves on the measured $R\left(q_{z}\right)$. This is demonstrated in Fig. 3 where we plot the background-subtracted and incident-intensitynormalized intensity scattered by the surface capillary waves measured as a function of $\beta$ for a fixed incidence angle $\alpha$ in the reflection plane $\left(\Delta \Theta=0^{\circ}\right)$. The agreement between the measured values (points) and the theoretical cross section (line) is very good over more than three decades in intensity.

For this scan the incident beam is $100 \mu \mathrm{m}$ high and the detector is $0.5 \mathrm{~mm}$ high and $1 \mathrm{~mm}$ wide. Integration of the intensity in this figure over the $\beta$ range spanned by the resolution function of the reflectivity measurements shown in Fig. 2 yields a value identical with that obtained in the reflectivity measurements at $q_{z}=0.48 \AA^{-1}$. This further strengthens our claim that the effects of the capillary-wave scattering on the reflectivity are well understood and can be separated out confidently from the measured $R\left(q_{z}\right)$.

The iterative procedure by which the theoretical line in Fig. 3 is calculated is the following. We first obtain a measure of the structure factor from a best fit of Eq. 1 to

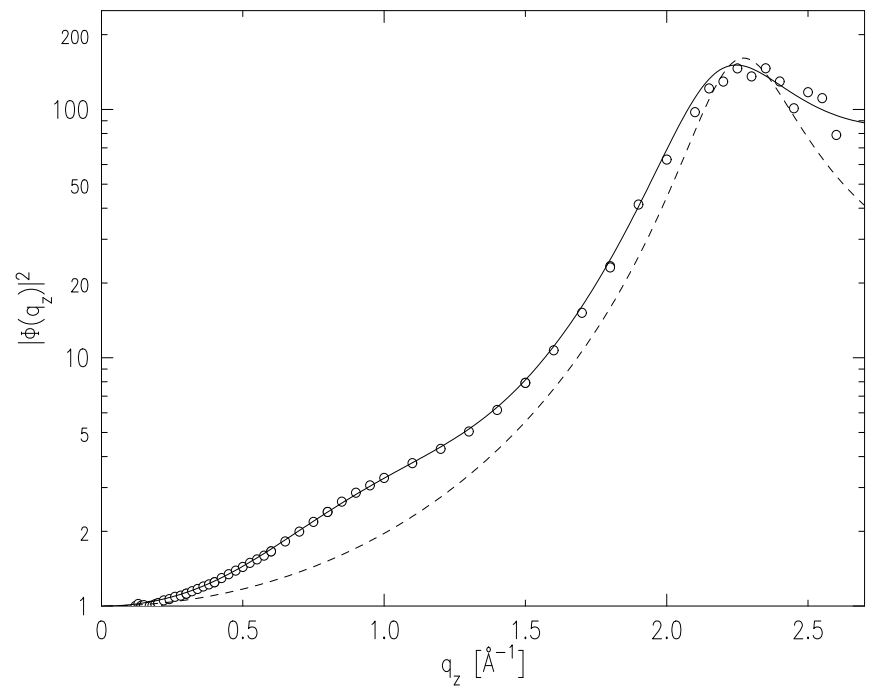

FIG. 4: The structure factor (squared) of the Sn surface as derived from the measured reflectivity $\left|\Phi\left(q_{z}\right)\right|^{2}=$ $R\left(q_{z}\right) /\left[R_{F}\left(q_{z}\right) C W\left(q_{z}\right)\right]$ (open circles). The dashed line is the theoretically expected $\left|\Phi\left(q_{z}\right)\right|^{2}$ of a simple layered density profile as found previously for Ga and In. The solid line is a fit to a model, discussed in the text, where the distance between the first and second layers is reduced by $10 \%$ relative to that of the subsequent layers.

the reflectivity (Fig. 2) using values for $\mathrm{CW}\left(q_{z}\right)$ that are calculated from published values for the surface tension. This form of the surface structure factor is then used to fit measured diffuse scattering curves similar to that shown in Fig. 3 with the surface tension as the only adjustable parameter. When needed, the cycle can be repeated using now the new surface tension value. In practice, however, a single cycle was enough, since the best fit value for the surface tension, $\gamma=560 \mathrm{mN} / \mathrm{m}$, was in good agreement with the published values. Thus, we assert that the form of the diffuse scattering that gives rise to the value of $C W\left(q_{z}\right)$ is fully determined, and the only unknown quantity in Eq. 1 is the surface structure factor. This quantity is the Fourier transform of the surface-normal derivative of the local electron density $\rho(z): \mathbb{1 5}^{15}$

$$
\Phi\left(q_{z}\right)=\frac{1}{\rho_{\infty}} \int d z \frac{d\langle\rho(z)\rangle}{d z} \exp \left(\imath q_{z} z\right)
$$

where $\rho_{\infty}$ is the electron density of the bulk and $\langle\ldots\rangle$ denotes averaging over the surface-parallel $(x, y)$ directions.

The ratio $R\left(q_{z}\right) /\left[R_{F}\left(q_{z}\right) C W\left(q_{z}\right)\right]=\left|\Phi\left(q_{z}\right)\right|^{2}$ derived from the measured reflectivity, is plotted in Fig. 田(open circles). The dashed line is the theoretical reflectivity calculated using the surface structure factor obtained from the simple atomic layering model that successfully described the intrinsic surface structure factor of the pure liquid $\mathrm{Ga}$ and In. As can be seen from the plot, this model does not describe the measured values well. In particular, it does not exhibit any feature corresponding to the weak but distinct shoulder observed in the measured values at $q_{z} \approx 0.9 \AA^{-1}$. As this shoulder's 


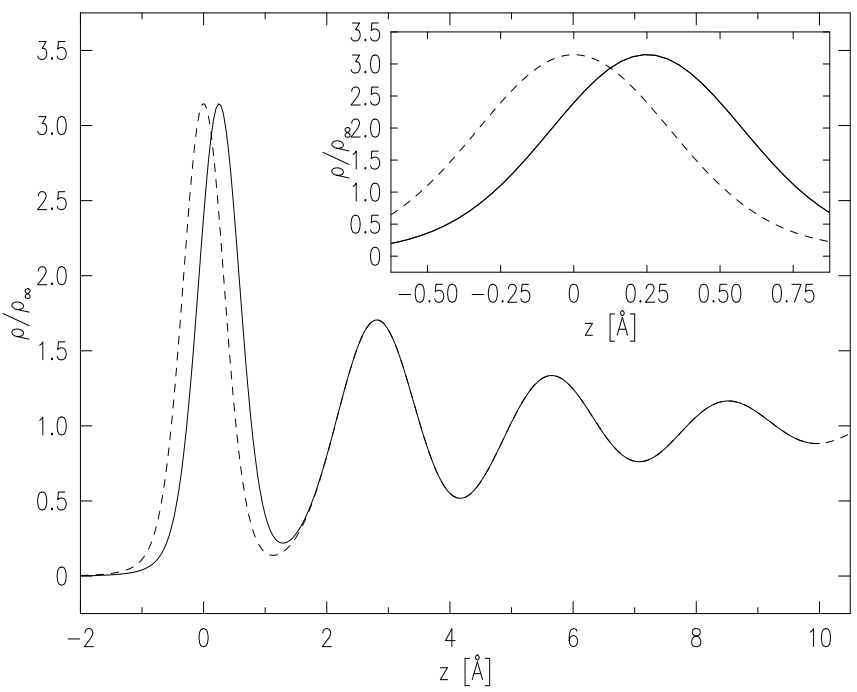

FIG. 5: Models for the intrinsic surface-normal electron density profiles of Sn for a simple, equally spaced layering model (dashed line), and for a model including a contraction of the spacing between the first and second layers by $10 \%$ (solid line). The models yield the reflectivities shown by the same lines in Fig. 2] The inset is a blow up of the surface layer region.

position is incommensurate with the large layering peak at $2.2 \AA^{-1}$, it must indicate the existence of a second length scale, additional to the periodicity of the surfaceinduced layering. It is highly unlikely that this second length scale $2 \pi / 0.9=6.96 \AA$ is associated with a periodic structure, in the same way that the $2.2 \AA^{-1}$ peak is associated with the surface-induced layering, since such two-periodicity structure would be very difficult to rationalize physically. Similarly, the low $q_{z}$ shoulder cannot be assigned to just a single-periodicity layered structure with a top layer which is denser than the subsequent layers. While such a structure will yield a subsidiary peak at low $q_{z}$, this peak would be commensurate with the high- $q_{z}$ one, i.e. appear at $q_{z}=0.5 \times 2.2 \approx 1.1 \AA^{-1}$ rather than the observed $\sim 0.9 \AA^{-1}$. Additionally, our attempts to fit the data using alternative density models - such as keeping the periodicity intact while modifying electron densities of the top several layers - did not produce a satisfactory fit to the experimental reflectivity data. Thus, we conclude that an appropriate model needs to include a second, noncommensurate and nonperiodic length scale. The solid line shown in Fig. 4 is a fit of the measured data by a model constructed along these lines. As can be seen, a good agreement is achieved with the measured values. We now proceed to discuss the details of this model.

The simple density model that has been used to represent the reflectivity of $\mathrm{Ga}^{3}$ and $\mathrm{In}^{4}$ consists of a convolution of an intrinsic density profile and a Debye-Waller-like factor, which accounts for the smearing of this profile by the thermally excited capillary waves. The intrinsic profile is modeled by a semi-infinite series of equally spaced
Gaussians, each representing a single atomic layer. The Gaussians have equal integrated areas (i.e. equal areal electron density) but their widths increase with depth below the surface. This, in turn, results in a gradual decrease with depth of the individual peaks and valleys of the density profile, and an eventual evolution of the density towards the constant average density of the bulk liquid. The decay length of the surface layering is typically of the order of just a few atomic spacings 3.4 The density profile of this model is shown in a dashed line in Fig. [5 and yields the dashed line in Fig. 4. The solid line that runs through the measured values in Fig. 4 is calculated from a slightly modified model, shown in a solid line in Fig. 5] The only difference between this model and the original one is that the distance between the first and second layers, $2.55 \AA$, is smaller by $\sim 10 \%$ than the $2.8 \AA$ spacing of the subsequent layers. The average density over the first two layers is thus larger than that of the bulk, and a second, nonperiodic, length scale is introduced. The good agreement of this minimallymodified model with the measured data, demonstrated in in Fig. 4 strongly supports our interpretation of the surface structure on liquid Sn.

\section{THE CASE FOR EXCLUSION OF SURFACE IMPURITIES}

Up to this point we have not considered the possibility that the dense surface layer may be a layer of atoms of a different metal adsorbed onto the Sn surface. This is precisely what happens, for example, in the liquid binary alloys $\mathrm{GaBi}^{13,16}$ and $\mathrm{Ga}-\mathrm{Pb}, \stackrel{17}{ }$, where a (dense) monolayer of the lower-surface-energy species $(\mathrm{Bi}, \mathrm{Pb})$ is found to Gibbs-adsorb at the free surface of the alloy. We now show experimental evidence that this is not the case here.

Consideration of the Gibbs rule ${ }^{18}$ shows that relatively low concentrations of three metals, $\mathrm{Bi}(\gamma=378 \mathrm{mN} / \mathrm{m}$, $\left.\rho_{e}=2.49 \mathrm{e} / \AA^{3}\right), \mathrm{Pb}\left(\gamma=458 \mathrm{mN} / \mathrm{m}, \rho_{e}=2.63 \mathrm{e} / \AA^{3}\right)$ and $\mathrm{Tl}\left(\gamma=464 \mathrm{mN} / \mathrm{m}, \rho_{e}=2.79 \mathrm{e} / \AA^{3}\right)$ could have produced surface electron densities sufficient to cause the low- $q_{z}$ shoulder shown in Fig. 4 In order to address this possibility we performed two independent experiments, grazing incidence x-ray fluorescence (GI-XRF) and resonant x-ray reflectivity, both of which are sensitive to the presence of sub monolayer quantities of impurities at the surface. None of the two measurements found evidence for contamination of the liquid Sn surface by a foreign species. Therefore, this supports the conclusion that the anomalous density feature observed here is in fact an intrinsic property of the liquid Sn surface.

\section{A. Grazing incidence fluorescence scattering}

The experimental setup used in these measurements is shown in Fig. 6] The sample was illuminated by $\mathrm{x}$ rays of wavelength $\lambda=0.42 \AA(\mathrm{E}=29.5 \mathrm{keV})$, in- 


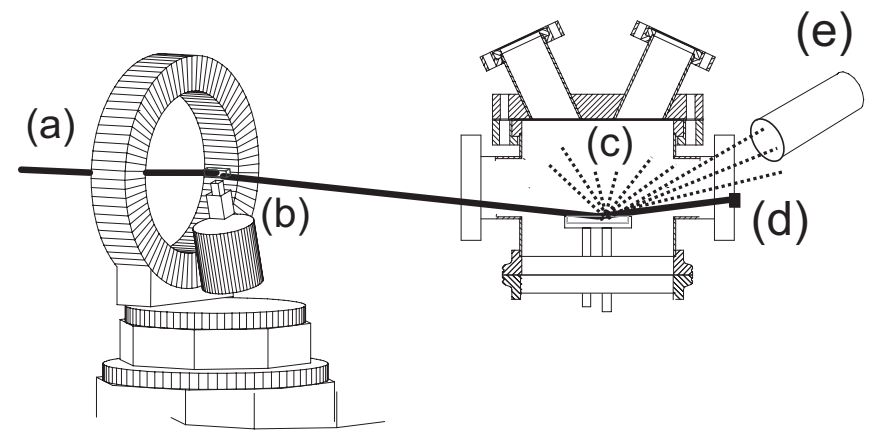

FIG. 6: Experimental setup used in the GI-XRF measurements: the synchrotron beam (a) is Bragg-reflected from a monochromator (b) which directs it downwards at the liquid Sn sample located inside a UHV chamber (c). The specularly reflected beam (solid line) is blocked by a beam stop on the exit window (d) to minimize the background. The fluorescence from the sample's surface (dotted lines) is detected off specularly by an energy-dispersive detector (e) located as close to the chamber as possible to maximize its solid angle of acceptance.

cident at a grazing angle of $\alpha=0.03^{\circ}$. Since this is approximately one-third of the critical angle of Sn at this energy, the refracted wave is evanescent. Its penetration depth below the surface is given by ${ }^{19} \tau=$ $1 / \operatorname{Im}\left[(4 \pi / \lambda) \sqrt{\alpha^{2}-\alpha_{c r i t}^{2}-\imath \lambda \mu / 2 \pi}\right]$, where the critical angle for $\mathrm{Sn}$ is $\alpha_{\text {crit }}=0.09^{\circ}$ and the linear absorption coefficient is $\mu=3.26 \times 10^{-6} \AA^{-1}$. For these values the incident beam probes only the uppermost $\tau=30 \AA$ of the sample. Due to the high surface tension, the surface of the liquid Sn sample is curved, which has to be taken into account as well: for an incident beam height $\mathrm{H}$ and a convex sample with a radius of curvature $r \approx 10 \mathrm{~m}$ the angle of incidence $\alpha$ varies over the illuminated area by $\sim H /(\alpha r)$. In these measurements $H$ was set to $5 \mu \mathrm{m}$, so that for a nominal angle of incidence $\alpha_{0}=0.03^{\circ}$ the local incidence angle varies by slightly less than $\pm 0.03^{\circ}$. Over this range, $0^{\circ}<\alpha<0.06^{\circ}$, the penetration length $\tau$ varies from $21 \AA$ to $28 \AA$. Thus, for our nominal incident angle of $\alpha \approx 0.03^{\circ}$ any detected fluorescence signal comes mostly from the top 8-10 atomic layers. Depending on the signal-to-noise ratio and other factors, such as the relative fluorescence yield, with this geometry it is possible to detect trace amounts of selected materials to sub-monolayer accuracies, as we show below. Note that at our incident energy, $29.5 \mathrm{keV}$, which is well above the Sn K edge, the K lines of Sn are all excited. However, the $\mathrm{K}$ edges of $\mathrm{Bi}, \mathrm{Pb}$ and $\mathrm{Tl}$ are all $>29.5 \mathrm{keV}$, so that only the L lines of these elements are excited.

Fluorescent x-ray emission from the illuminated portion of the sample was detected by an energy dispersive intrinsic Ge detector (area $\sim 9 \mathrm{~mm}^{2}$ ) that was mounted about $30 \mathrm{~cm}$ away from the center of the sample, $15 \mathrm{~cm}$ above it, and displaced azimuthally by about $30^{\circ}$ from the incidence plane. The experimental setup for this geometry is shown in Fig. [6] The signal was recorded using

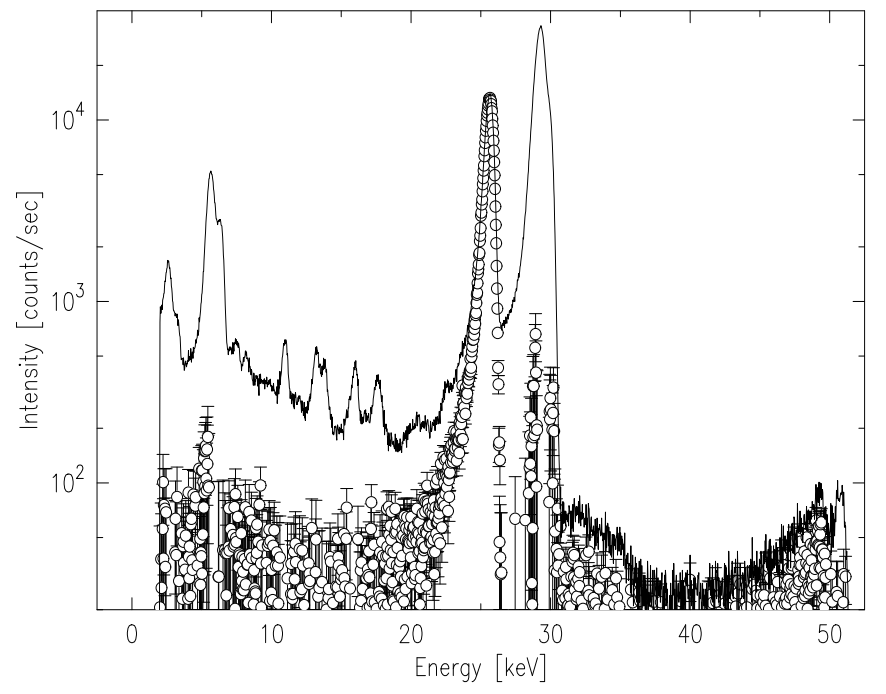

FIG. 7: Raw (line) and background-subtracted (open circles) measured fluorescence from the surface of liquid Sn. The sharp lines are due to the $\mathrm{K}$ emissions lines ${ }^{20}$ of $\mathrm{Fe}$ $(\mathrm{E}=6.4 \mathrm{keV}), \mathrm{Nb}(\mathrm{E}=16.6 \mathrm{keV}), \mathrm{Mo}(\mathrm{E}=17.5 \mathrm{keV})$ and $\mathrm{Sn}$ $(\mathrm{E}=25.2 \mathrm{keV})$.

a multichannel analyzer. A lead shield was mounted on the output window as shown in the Fig. [6 to eliminate scattering from the specularly reflected beam into the detector by the exit window or outside air. Unfortunately, there was no simple way to shield the detector from scattering or fluorescence originating in the entrance window of the UHV chamber. In order to account for this background scattering, a differential measurement has been performed. The signal detected with the sample displaced $3 \mathrm{~mm}$ below the incident beam was subtracted from the signal that was measured for the beam striking the sample. The fluorescence data are shown in Fig. 7 The solid line is the as-measured raw fluorescence spectrum. It consists of fluorescence from both the sample and the entrance window, elastic scattering from the incident beam (the strongest peak at $\sim 29.15 \mathrm{keV}$ ), and $\mathrm{Sn}$ fluorescence (at $25.2 \mathrm{keV}$ ). There are a number of lowerenergy peaks originating in the UHV chamber's components. The open circles show the spectrum obtained after subtracting from the raw spectrum the background spectrum recorded when the sample is moved out of the beam. The only remaining prominent peak is the Sn fluorescence observed at $25.2 \mathrm{keV}$. No other characteristic lines are discernible above the noise level. The ratio of the Sn K-fluorescence to the noise level in the 10-15 keV energy region, where the L-fluorescence lines of the $\mathrm{Pb}$, $\mathrm{Bi}$ or $\mathrm{Tl}$ are expected, is $\sim 200$. Factoring in the ratio of the K fluorescence yield of Sn (0.84) to the L-fluorescence yields of $\mathrm{Pb}, \mathrm{Bi}$ or $\mathrm{Tl}(\sim 0.4)$ indicates that the presence of as little as $10 \%$ of a full monolayer of these metals (out of the $\sim 10$ atomic layers of Sn illuminated by the evanescent wave) should be detectable in this experiment. Since an impurity coverage of the surface required to generate the $\sim 0.9 \AA^{-1}$ reflectivity peak is significantly higher than 
that, the absence of an impurity signal in Fig. 7 rules out with a high degree of confidence the possibility that a Gibbs-adsorbed layer of impurities is the originator of the $0.9-\AA^{-1}$ feature.

\section{B. Resonant x-ray reflectivity}

The effective electron density of an x-ray-scattering atom is proportional to the scattering form factor $f(q)+$ $f^{\prime}(q, E)$. Here $f(q) \rightarrow Z$ for $q \rightarrow 0$ and $Z$ is the atomic number. When the x-ray energy is tuned through an absorption edge of a scattering atom, the magnitude of the real part of $f^{\prime}$ undergoes a sharp decrease, producing a change in the scattering power of that specific atom. ${ }^{21,22}$ Thus, by measuring the scattering on and off edge it is possible to isolate the scattering due to the specific atom. We have used this method, called resonant (or anomalous) scattering, in the reflectivity mode to probe the liquid surface of $\mathrm{Sn}$ for the presence of foreign atoms. This is done by comparing the x-ray reflectivities of the liquid surface measured with the $\mathrm{x}$-ray energy tuned on and off the $\mathrm{K}$ edge of $\mathrm{Sn}(29.20 \mathrm{keV})$. If the low $q_{z}$ feature at $q_{z} \approx 0.9 \AA^{-1}$ is due a thin surface layer of foreign atoms, the effective electron density of Sn at the edge will change, while that of the foreign atoms will not. Thus, the density contrast will change and so will the prominence of the low $-q_{z}$ feature. If, however, the low- $q_{z}$ shoulder is due to an intrinsic Sn structure, the electron density difference between high-density surface layer and the bulk will remain unchanged and so will the low- $q_{z}$ shoulder.

The $29.20 \mathrm{keV}$ edge of Sn was identified by a transmission measurement through a $\mathrm{Sn}$ foil, shown in Fig. 8(b), as well as by the reflectivity from the liquid Sn surface at a fixed $q_{z}=0.3 \AA^{-1}$, shown in Fig. 8(a). Since the surface structure factor $\Phi\left(q_{z}\right)$ depends on the electron density contrast between surface and bulk, the only energy dependence of $R\left(q_{z}\right)$ away from both structural peaks ( at $q_{z}=0.9 \AA^{-1}$ and $q_{z}=2.3 \AA^{-1}$ ) is due to the energy variation of the critical wave vector $q_{c}$. This leads to a $\left(Z+f^{\prime}(E)\right)^{2}$ energy dependence of the reflectivity at a fixed $q_{z}$. The minimum value of $f^{\prime}(E)$, estimated from Fig. 8 (a), is consistent with the theoretically predicted value calculated by the IFEFFIT $^{23}$ program, taking the smearing due to the lifetime widths of the $\mathrm{K}$ and $\mathrm{L}$ levels into consideration. The observed change in reflectivity at the minimum relative to that $50 \mathrm{eV}$ away is measured to be about $15 \%$, which corresponds, in turn, to a $7 \%$ reduction in the effective electron density of $\mathrm{Sn}$.

In Fig. 9 we show the low $-q_{z}$ normalized reflectivity data $R\left(q_{z}\right) /\left[R_{F}\left(q_{z}\right) C W\left(q_{z}\right)\right]=\left|\Phi\left(q_{z}\right)\right|^{2}$, measured at the $\mathrm{K}$ edge of $\mathrm{Sn}(29.20 \mathrm{keV})$, just above the edge $(29.22$ $\mathrm{keV})$ and far below the edge $(17 \mathrm{keV})$. The fact that the three data sets coincide proves unambiguously that the $0.9-\AA^{-1}$ reflectivity feature does not arise from contrast between $\mathrm{Sn}$ and a different element. A more quantitative analysis can be done in two ways. First, we as-

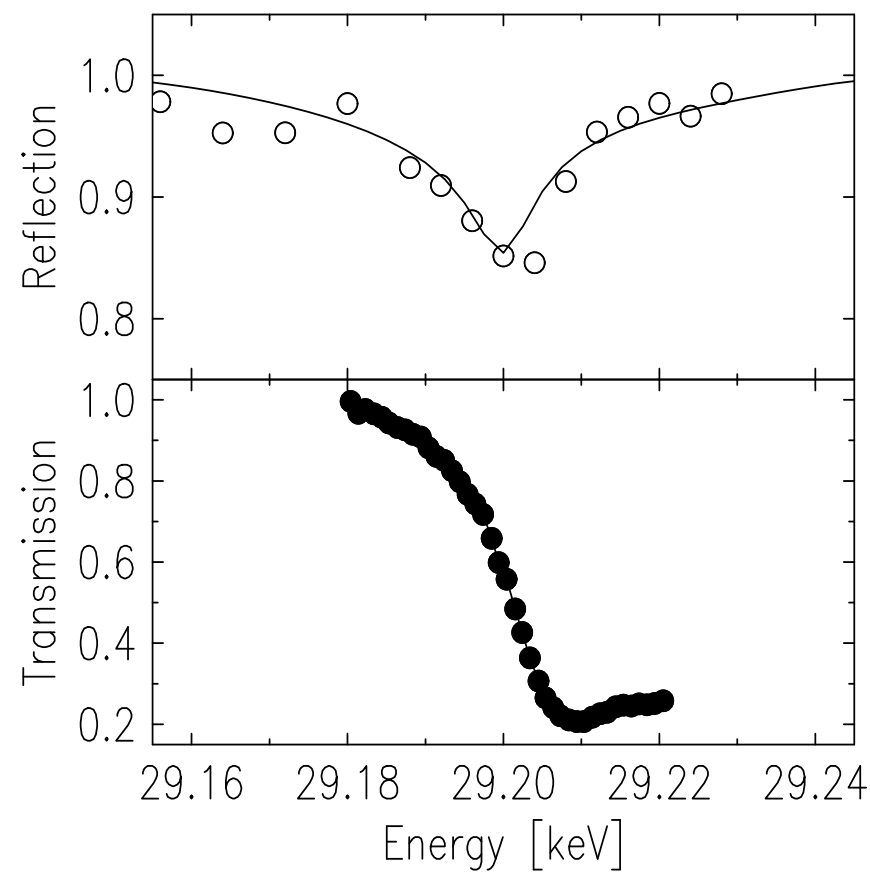

FIG. 8: (Top) Relative energy variation of the reflectivity off the liquid Sn surface at a fixed $q_{z}=0.3 \AA^{-1}$. The line is the theoretical prediction of the change in the reflectivity due to the variation of the dispersion correction $f^{\prime}(E)$ of the scattering factor near the edge, as calculated by the IFEFFIT ${ }^{23}$ program. The open circles are the measured values. (Bottom) Measured energy variation of the intensity transmitted through an Sn foil (points). The K edge of Sn at E=29.20 keV shows up clearly in both measurements.

sume a simple box model for the surface, 19 shown in the inset to Fig. 9. In this model, the surface layer's electron density $\mathrm{A}$ is larger than that of the bulk, B, yielding $\left|\Phi\left(q_{z}\right)\right|^{2} \approx(2 A / B-1)^{2}$ at the peak. To obtain the measured peak of $\left|\Phi\left(q_{z}\right)\right|^{2}=1.4$, we have to assume $A / B=1.1$. We note that only a handful of elements would be consistent with a model where this $10 \%$ increase in density originates in a surface layer of impurity atoms. Eliminating the elements which have a higher surface tension than that of $\mathrm{Sn}(\gamma=560 \mathrm{mN} / \mathrm{m})$, which will not Gibbs-segregate at the surface, the only reasonably likely remaining candidates are $\mathrm{Bi}, \mathrm{Pb}$ and Tl. If we assume that the surface layer is a monolayer of any one of these elements, the effect of the resonance is that the effective electron density of the Sn would be reduced by about $7 \%$, as mentioned above. The measured $R\left(q_{z}\right) /\left[R_{F}\left(q_{z}\right) C W\left(q_{z}\right)\right]=\left|\Phi\left(q_{z}\right)\right|^{2}$ would therefore increase at the Sn K-edge to $(2 \times 1.1 / 0.93-1)^{2}=1.86$. The fact that this does not occur proves, again, that the increased density at the surface is an intrinsic surface effect, due entirely to a structure comprising Sn atoms only.

A second quantitative argument is the full modeling of the reflectivity, rather than considering only the maximum of the $0.9-\AA^{-1}$ peak. The solid line in Fig. 9] is 


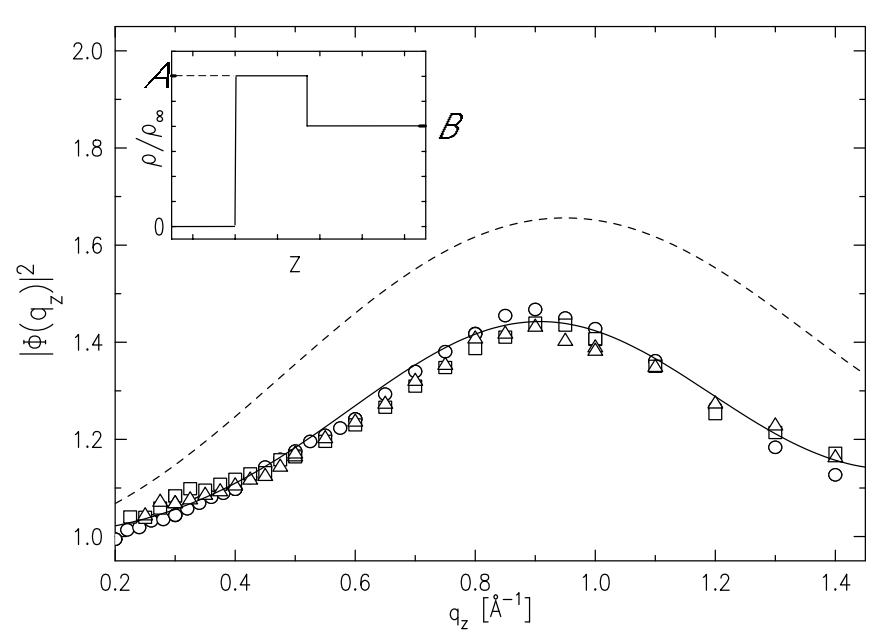

FIG. 9: The structure factor (squared) of the Sn surface as derived from the measured reflectivity, at $(\mathrm{E}=29.20 \mathrm{keV}$, triangles), above $(\mathrm{E}=29.22 \mathrm{keV}$, circles $)$ and below $(\mathrm{E}=17 \mathrm{keV}$, squares) the K-edge. The solid line is a fit to the density model featuring reduced interatomic spacing for the top layer by approximately $10 \%$. The dashed line is calculated for a model with a high-density surface monolayer of an atomic species other than Sn. The inset shows the density profile of such a layer. For a discussion see the text.

the same fit shown in Fig. 5 to the model where the density of each atomic layer is kept at the Sn density value, while the spacing between the first and second atomic layers is reduced from the $2.80 \AA$ of subsequent layers to $2.55 \AA$. The agreement of all measured reflectivities, in particular that measured at the edge (triangles), with this model is excellent. The dashed line is the reflectivity calculated assuming an additional surface monolayer of a different species having a density higher than that of Sn. As can be seen, for the on-edge measurement, this line is expected to lie considerably higher than off edge. It clearly does not agree with the on-edge measured data (triangles).

The results of the two measurements presented in this section rule out the possibility that the dense surface layer found in the reflectivity measurements is due to surface segregation of impurity atoms. Thus, they strongly supports our claim that the high-density layer present at the surface is an intrinsic property of Sn itself.

\section{CONCLUSION}

We have found that the surface of liquid Sn exhibits atomic layering, similar to that found in other metallic systems. However, the surface structure factor exhibits an additional low- $q_{z}$ shoulder, which has not been observed for any of the pure metals studied to date. The possibility that this structure is due to a layer of surfaceadsorbed contaminants has been addressed by two experiments, both of which confirm that the observed reflectivity feature is an intrinsic property of liquid Sn. The strongest argument comes from the resonant x-ray reflectivity measurement which finds no detectable difference between reflectivities measured on and off the Sn K edge, while model calculations predict a significant change for a layer of foreign surface atoms. Our analysis shows that the anomalous feature at low $q_{z}$ is consistent with a modified atomic layering density model where the spacing between the first and second atomic layers is reduced by $10 \%$ relative to the subsequent ones, thereby increasing the average density at the surface.

The physical reasons for the existence of this denser layer at the surface of liquid Sn are not clear, since the studies of other liquid metals and alloys exhibit no evidence for such a feature. It should be noted that the observed spacing reduction is similar to the well-known surface relaxation phenomena found in many crystalline monatomic metals. Surface relaxation typically manifests itself in a reduction of the lattice spacing between the surface atomic layer and first subsurface atomic layer commonly of the order $5 \%$ to $10 \% 24$, which is comparable to the spacing reduction found in this study. Additionally, surface relaxation is generally enhanced for the metals exhibiting a high degree of covalent bonding 25 , while Sn-Sn pairing is well known to be present in the bulk phase $\frac{10,11,12}{0}$ of liquid Sn. And since contrary to solids the surface reconstruction mechanism is not possible at liquid surfaces due to absence of outward-oriented dangling bonds at the surface layer, surface relaxation remains as the primary mechanism of minimizing the surface energy associated with a reduced number of nearest neighbors at the surface.

Another argument to consider comes from Sn having a rather complicated crystalline structure below melting point: the density of the liquid $\mathrm{Sn}\left(6970 \mathrm{~kg} / \mathrm{m}^{3}\right)$ is in between the densities of the solid $\alpha-\operatorname{Sn}\left(5750 \mathrm{~kg} / \mathrm{m}^{3}\right)$, also known as "gray tin" and forming a cubic atomic structure, and the solid $\beta$-Sn $\left(7310 \mathrm{~kg} / \mathrm{m}^{3}\right)$, known as "white tin" and forming a tetragonal atomic structure. It is possible that while the higher-density $\beta$ phase is no longer stable in the bulk beyond the melting point, it prevails in some form at the surface, where the packing restrictions are relaxed due to a smaller number of nearest neighbors.

\section{ACKNOWLEDGMENTS}

We thank Matt Newville, Larry Sorensen, and John Rehr for helpful discussions on IFEFFIT use. This work has been supported by U.S. Department of Energy Grant No. DE-FG02-88-ER45379, National Science Foundation Grant No. DMR-01-12494, and the U.S.-Israel Binational Science Foundation, Jerusalem. ChemMatCARS Sector 15 is principally supported by the National Science Foundation/Department of Energy under Grant No. CHE0087817. The Advanced Photon Source is supported by the U.S. Department of Energy, Basic Energy Sciences, Office of Science, under Contract No. W-31-109Eng-38. 
1 S.A. Rice, D. Guidotti, and H.L. Lemberg. Aspects of the study of surfaces, (Wiley, Chichester, UK, 1974) vol. 27, pp. 543-633.

2 O. M. Magnussen, B. M. Ocko, M. J. Regan, K. Penanen, P. S. Pershan, and M. Deutsch. Phys. Rev. Lett. 74, 4444 (1995).

3 M. J. Regan, E. H. Kawamoto, S. Lee, P. S. Pershan, N. Maskil, M. Deutsch, O. M. Magnussen, B. M. Ocko, and L. E. Berman Phys. Rev. Lett. 75, 2498 (1995).

${ }^{4}$ H. Tostmann, E. DiMasi, P. S. Pershan, B. M. Ocko, O. G. Shpyrko, and M. Deutsch. Phys. Rev. B 59, 783 (1999).

5 O. Shpyrko, P. Huber, A. Grigoriev, P. Pershan, B. Ocko, H. Tostmann, and M. Deutsch Phys. Rev. B 67, 115405 (2003).

6 E. Dimasi, H. Tostmann, B. M. Ocko, P. S. Pershan and M. Deutsch Phys. Rev. B 58, 13419 (1998).

7 A. Di Cicco and A. Filliponi Europhys. Lett. 27, 407 (1994).

8 X. S. Gong et al. Europhys. Lett. 21, 469 (1994).

9 W. J. Huisman, J. F. Peters, M. J. Zwanenburg, S. A. deVries, T. E. Derry, D. Abernathy, J. F. van der Veen Nature 390, 379 (1997).

10 D. Jovic and I. Padureanu. Journal of Physics C 9, 1135 (1976).

11 A. DiCicco. Phys. Rev. B 53, 6174 (1996).

12 T. Itami, S. Munejiri, T. Masaki, H. Aoki, Y. Ishii, T. Kamiyama, Y. Senda, F. Shimojo, and K. Hoshino. Phys. Rev. B 67, 064201 (2003).

13 P. Huber, O. G. Shpyrko, P. S. Pershan, B. M. Ocko, E. DiMasi, and M. Deutsch Phys. Rev. Lett. 89, 035502 (2002).
14 O. G. Shpyrko, M. Fukuto, B. M. Ocko, P. S. Pershan, M. Deutsch, T. Gog and I. Kuzmenko. Phys. Rev. B 69, 245423, (2004).

15 A. Braslau, P. S. Pershan, G. Swislow, B. M. Ocko, and J. Als-Nielsen. Phys. Rev. A 38, 2457 (1988).

16 H. Tostmann, E. DiMasi, O. G. Shpyrko, P. S. Pershan, B. M. Ocko, and M. Deutsch. Phys. Rev. Lett. 84, 4385 (2000).

17 B. Yang, D. Li, Z. Huang, and S. A. Rice Phys. Rev. B 62, 13111 (2000).

18 J. W. Gibbs, R. G. Van Name, W. R. Longley, and H. A. Bumstead, Collected works of J. Willard Gibbs, (Longmans, Green and Co., New York, 1928).

19 M. Tolan. X-Ray Scattering from Soft-Matter Thin Films, (Springer-Verlag New York, 1999) p. 148.

20 J. A. Bearden. Rev. Mod. Phys., 39, 78-124 (1967).

21 C. J. Sparks and K. Fischer. Resonant Anomalous X-ray Scattering: Theory and Applications, (North-Holland, Amsterdam, 1994).

22 E. DiMasi, H. Tostmann, O. G. Shpyrko, M. Deutsch, P. S. Pershan, and B. M. Ocko. Journal of Physics: Condensed Matter 12, 209 (2000).

${ }^{23}$ Matt Newville IFEFFIT: Interactive XAFS Analysis, http://cars9.uchicago.edu/ifeffit/ 1997.

24 C. Kittel, Introduction to Solid State Physics, Wiley, New York, 1996.

${ }^{25}$ K. Kaddas, S. Alvarez, E. Ruiz, P. Alemany, Phys. Rev. B 53, 4933 (1996). 